\title{
Intrinsic Functional Boundaries of Lateral Frontal Cortex in the Common Marmoset Monkey
}

\author{
David J. Schaeffer, ${ }^{1}$ Kyle M. Gilbert, ${ }^{1}$ Joseph S. Gati, ${ }^{1}$ Ravi S. Menon, ${ }^{1,2}$ and Stefan Everling ${ }^{1,2}$ \\ ${ }^{1}$ Centre for Functional and Metabolic Mapping, Robarts Research Institute, and ${ }^{2}$ Department of Physiology and Pharmacology, University of Western \\ Ontario, London, Ontario N6A 5B7, Canada
}

The common marmoset (Callithrix jacchus) is a small New World primate species that has been recently targeted as a potentially powerful preclinical model of human prefrontal cortex dysfunction. Although the structural boundaries of frontal cortex were described in marmosets at the start of the 20th century (Brodmann, 1909) and refined more recently (Paxinos et al., 2012), the broad functional boundaries of marmoset frontal cortex have yet to be established. In this study, we sought to functionally derive boundaries of the marmoset lateral frontal cortex (LFC) using ultra-high field (9.4 T) resting-state functional magnetic resonance imaging (RS-fMRI). We collected RS-fMRI data in seven (four females, three males) lightly anesthetized marmosets and used a data-driven hierarchical clustering approach to derive subdivisions of the LFC based on intrinsic functional connectivity. We then conducted seed-based analyses to assess the functional connectivity between these clusters and the rest of the brain. The results demonstrated seven distinct functional clusters within the LFC. The functional connectivity patterns of these clusters with the rest of the brain were also found to be distinct and organized along a rostrocaudal gradient, consonant with those found in humans and macaques. Overall, these results support the view that marmosets are a promising preclinical modeling species for studying LFC dysfunction related to neuropsychiatric or neurodegenerative human brain diseases.

Key words: lateral frontal cortex; marmoset; resting-state fMRI; ultra-high field

\section{Significance Statement}

The common marmoset is a New World primate that has garnered recent attention as a powerful complement to canonical Old World primate (e.g., macaques) and rodent models (e.g., rats, mice) for preclinical modeling of the human brain in healthy and diseased states. A critical step in the development of marmosets for such models is to characterize functional network topologies of frontal cortex in healthy, normally functioning marmosets, that is, how these circuitries are functionally divided and how those topologies compare to human circuitry. To our knowledge, this is the first study to demonstrate functional boundaries of the lateral frontal cortex and the corresponding network topologies in marmoset monkeys.

\section{Introduction}

The primate lateral frontal cortex (LFC) is critical for integrating neuronal processes that result in complex and flexible behavior (Fuster, 2001; Miller and Cohen, 2001; Tanji and Hoshi, 2008). With dysfunction of the LFC implicated across a range of neuropsychiatric disorders (e.g., schizophrenia; Shenton et al., 2001),

Received Oct. 6, 2018; revised Nov. 29, 2018; accepted Dec. 1, 2018.

Author contributions: D.J.S., K.M.G., J.S.G., R.S.M., and S.E. edited the paper; D.J.S., K.M.G., R.S.M., and S.E. designed research; D.J.S., K.M.G., J.S.G., and S.E. performed research; D.J.S. analyzed data; D.J.S. wrote the paper.

This work was supported by Canadian Institutes of Health Research Grant FRN 148365 and the Canada First Research Excellence Fund (to BrainsCAN). We thank Drs. Cirong Liu and Afonso Silva of the National Institutes of Health for providing the surface models of the marmoset brain. We also thank Nicole Hague, Miranda Bellyou, and Lauren Schaeffer for animal preparation and care and Dr. Alex Li for scanning assistance.

The authors declare no competing financial interests.

Correspondence should be addressed to Stefan Everling at severlin@uwo.ca.

https://doi.org/10.1523/JNEUROSCI.2595-18.2018

Copyright $\odot 2019$ the authors $\quad 0270-6474 / 19 / 391020-10 \$ 15.00 / 0$ the ability to model LFC dysfunction in nonhuman mammalian species is critical for investigating the etiology of these disorders. The common marmoset (Callithrix jacchus) is a small New World primate species that has been recently targeted as a potentially powerful preclinical model of human prefrontal cortex dysfunction (Okano et al., 2016). With a granular frontal cortex (Reser et al., 2017) and the advent of transgenic modification techniques (Sasaki et al., 2009; Park et al., 2016; Tomioka et al., 2017), marmosets are well positioned to serve as neuropsychiatric models of prefrontal cortex dysfunction (Okano et al., 2016). A critical step in the development of marmosets for such models, however, is to characterize functional network topologies of LFC that are homologous (and thus potentially comparable) with the human brain. Here, we sought to characterize the intrinsic functional boundaries of the LFC in marmosets using resting-state functional magnetic 
resonance imaging (RS-fMRI) in lightly anesthetized marmosets at ultra-high field $(9.4 \mathrm{~T})$.

Structurally, marmosets share the same basic architectonic organization of LFC with humans and macaques (Petrides, 2005; Paxinos et al., 2012; Reser et al., 2013). Although these regions share the same architectonic designations (Brodmann's areas 10, $9,46,47,8,45,6$, and 4 exist across the three species), the criteria to delineate the areas has varied across primate studies (for review, see Petrides, 2005). Furthermore, structural connectivity studies in humans and macaques suggest that fiber end points exhibit patterns that are distinct from those delineated through cytoarchitecture (Yeterian et al., 2012; Sallet et al., 2013). The functional boundaries of these regions also seem to differ, as it has been shown that the functional clusters of LFC may not strictly overlap with cytoarchitectonic boundaries (Sallet et al., 2013; Hutchison and Everling, 2014). As such, it is important to map the functional boundaries of these regions directly, rather than inferring function from structural organization.

RS-fMRI has allowed for functional mapping of broad LFC boundaries in both humans and macaques (Cohen et al., 2008; Goulas et al., 2012; Sallet et al., 2013; Hutchison and Everling, 2014). To date, however, little is known about the functional boundaries of LFC in marmosets. A clear benefit of using RSfMRI to map functional boundaries is that it is blind to cytoarchitectonic boundaries and thus not constrained by a priori hypotheses about LFC organization (e.g., those based on structural organization). Because RS-fMRI is task independent, it thus relieves the burden of task matching across species and the need for extensive training protocols in nonhuman primates. As such, RS-fMRI is well suited for identifying similarities or differences in integrated functional networks across primate species (e.g., between New and Old World primates).

In this study, we sought to functionally derive boundaries of marmoset LFC using RS-fMRI. We collected RS-fMRI in seven lightly anesthetized marmosets and used a data-driven hierarchical clustering approach to identify subdivisions of the LFC based on intrinsic functional connectivity. We then conducted seedbased analyses to assess the functional connectivity between these clusters and the rest of the brain. Given that marmosets are poised to become a leading transgenic primate model of preclinical human neuropsychiatric diseases (Sasaki et al., 2009; Kishi et al., 2014; Okano and Mitra, 2015; Okano et al., 2016), understanding the functional organization of LFC will be important as researchers leverage the phylogenetic similarity of marmosets to model such intractable diseases.

\section{Materials and Methods}

\section{Image acquisition}

Data were collected from seven (four females, three males) adult marmosets (Callithrix jacchus) aged 1-6 years and weighing 300-500 g. Before each imaging session, anesthesia was induced with ketamine hydrochloride at $20 \mathrm{mg} / \mathrm{kg}$. During scanning, marmosets were lightly anesthetized with isoflurane and maintained at a level of $1.5 \%$ throughout the scan by means of inhalation. Oxygen flow rate was kept between 1.75 and $2.25 \mathrm{~L} / \mathrm{min}$ throughout the scan. Respiration, blood oxygen saturation, and heart rate were continuously monitored via pulse oximeter and were observed to be within the normal range throughout the scans. Body temperature was also measured and recorded throughout, and was maintained using warm-water circulating blankets, thermal insulation, and warmed air. All animals were head-fixed in a stereotactic position using a custom-built MRI bed (Gilbert et al., 2018) with ear bars, eye bars, and a palate bar housed within the anesthesia mask (for the design, see Fig. 1). All imaging was performed at the Centre for Functional and Metabolic Mapping at the University of Western Ontario.
Experimental procedures were in accordance with the Canadian Council of Animal Care policy and a protocol approved by the Animal Care Committee of the University of Western Ontario Council on Animal Care.

Data were acquired using a $9.4 \mathrm{~T}, 31 \mathrm{~cm}$ horizontal bore magnet (Varian/Agilent) and Bruker BioSpec Avance III console with the software package Paravision-6 (Bruker BioSpin) and a custom-built, highperformance 15-cm-diameter gradient coil with $400 \mathrm{mT} / \mathrm{m}$ maximum gradient strength (xMR, London, Ontario, Canada; Peterson et al., 2018). A geometrically optimized eight-channel phased array receive coil was designed in-house for signal-to-noise (SNR) improvement and to allow for acceleration of the echoplanar imaging of marmoset cohorts (for open-source computer-aided design files, see Gilbert et al., 2018), with two open elements around the ear bars, which were electrically closed after the animal was head fixed via a brass screw (Fig. 1). Preamplifiers were located behind the animal, and the receive coil was placed inside a quadrature birdcage coil ( $12 \mathrm{~cm}$ inner diameter) used for transmission. Functional imaging was acquired during one session for each animal, with four to six functional runs (at 600 volumes each) with the following parameters: TR, $1500 \mathrm{~ms}$; TE, $15 \mathrm{~ms}$; flip angle, $35^{\circ}$; field of view, $64 \times 64 \mathrm{~mm}$; matrix size, $128 \times 128$; voxel size, $0.5 \times 0.5 \times 0.5 \mathrm{~mm}$; slices, 42; bandwidth, $500 \mathrm{kHz}$; generalized autocalibrating partial parallel acquisition (GRAPPA) acceleration factor, 2 (anterior-posterior). T2weighted structural scans were acquired for each animal with the following parameters: TR, $5500 \mathrm{~ms}$; TE, $53 \mathrm{~ms}$; field of view, $51.2 \times 51.2$ $\mathrm{mm}$; matrix size, $384 \times 384$; voxel size, $0.133 \times 0.133 \times 0.5 \mathrm{~mm}$; slices, 42; bandwidth, $50 \mathrm{kHz}$; GRAPPA acceleration factor, 2.

\section{Image preprocessing}

Data were preprocessed using Analysis of Functional NeuroImages (AFNI; Cox, 1996) and the FMRIB Software Library (FSL; Smith et al., 2004). Raw functional images were converted to Neuroimaging Informatics Technology Initiative (NIfTI) format using dcm2niix (Li et al., 2016) and reoriented from the sphinx position using FSL (for example raw functional volume, see Fig. 1). The images were then despiked (AFNI's 3dDespike) and volume registered to the middle volume (AFNI's 3 dvolreg). The motion parameters from volume registration were stored for later use with nuisance regression. Images were smoothed by a 1.5 $\mathrm{mm}$ full-width at half-maximum Gaussian kernel to reduce noise (AFNI's 3 dmerge). An average functional image was then calculated for each session and registered (FSL's FLIRT) to each animal's T2-weighted image. The $4 \mathrm{D}$ time series data were carried over using this transformation matrix. T2-weighted images were manually skull-stripped and this mask was applied to the functional images.

T2-weighted images were nonlinearly registered to the NIH marmoset brain atlas (Liu et al., 2018) using Advanced Normalization Tools (Avants et al., 2011), and the resultant transformation matrices were applied to the functional images. The olfactory bulb was manually removed from the T2-weighted images of each animal before registration, as it was not included in the template image.

\section{Experimental design and statistical analysis}

Hierarchical clustering. Once all images were in template space, the time series data were imported into MATLAB (The MathWorks) for hierarchical clustering analysis. Because we were explicitly interested in the LFC, we limited the clustering analysis to frontal areas $10,46 \mathrm{D}, 46 \mathrm{~V}, 47 \mathrm{~L}$, $9,8 \mathrm{~b}, 8 \mathrm{aD}, 8 \mathrm{aV}, 8 \mathrm{C}, 45,6 \mathrm{M}, 6 \mathrm{DR}, 6 \mathrm{DC}, 6 \mathrm{Va}, 6 \mathrm{Vb}, 4 \mathrm{ab}$, and $4 \mathrm{c}$ as defined in the Paxinos marmoset atlas (Paxinos et al., 2012), which is registered and included with the NIH template files (Liu et al., 2018). A crosscorrelation matrix between all voxels within the LFC mask (consisting of 1587 voxels) was calculated for each run using MATLAB's "partialcorr" function; explicitly, partial correlation coefficients were calculated between pairs of voxels within the time series matrix, while controlling for the remaining voxels within the matrix. Motion parameters, linear and nonlinear trends, and mean white matter time courses were used as nuisance regressors. Following a Fisher $z$-score transformation, the average $z$-score was calculated across scans (runs) within each animal, then the average across all animals was calculated. The result of this was the average pairwise correlation (i.e., one matrix) between all voxels within the LFC mask. Hierarchical clustering was then conducted to extract 
A

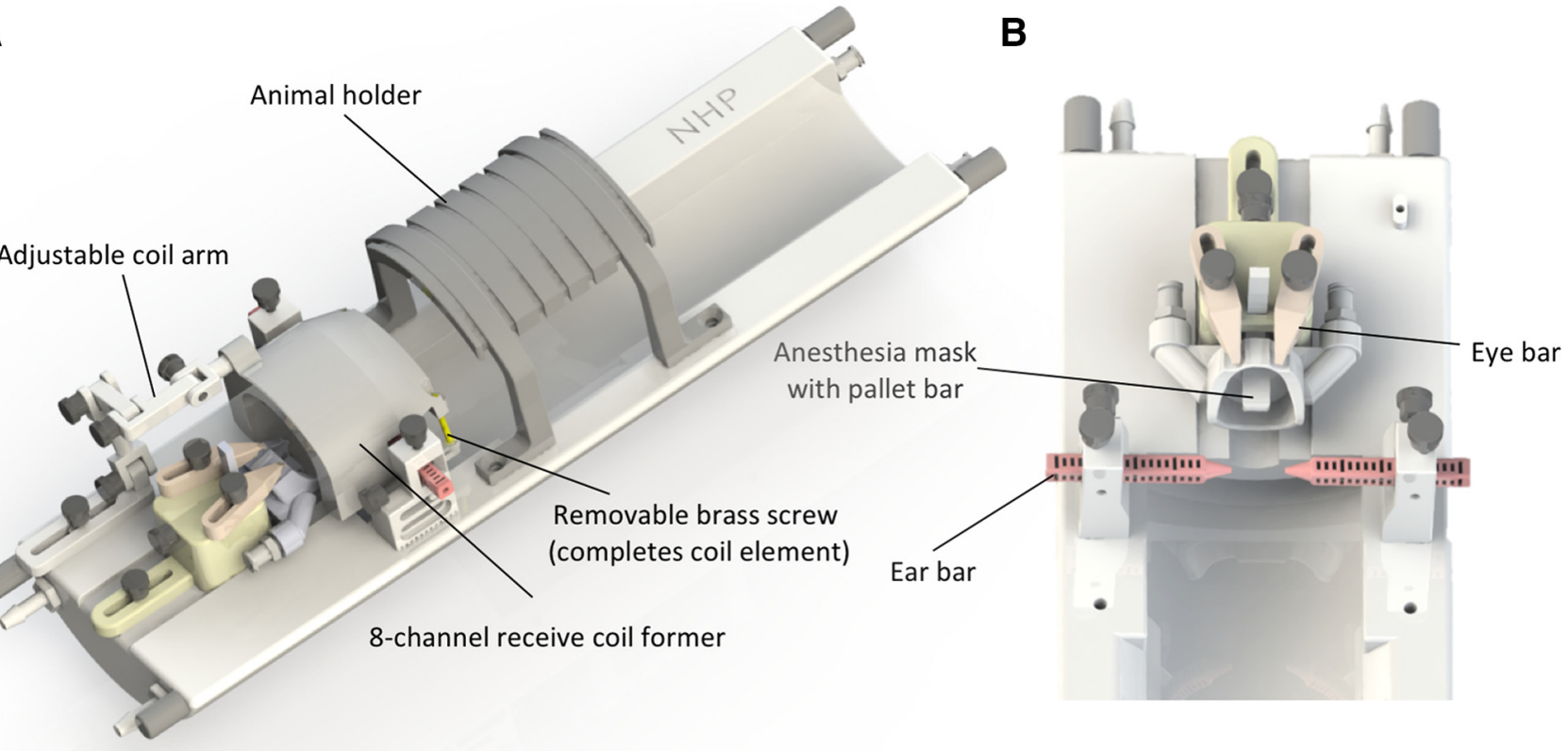

\section{c Raw EPI data}

\section{Group-wise temporal SNR}

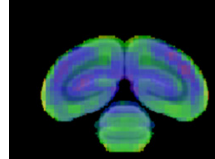

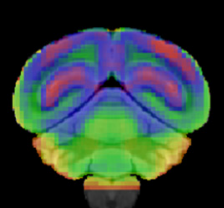

0

Temporal SNR

Figure 1. Computer-aided design (CAD) drawings of the stereotactic marmoset holder and radiofrequency coil former (in-house designs). $\boldsymbol{A}$, The marmoset tray with the radiofrequency coil installed via an adjustable coil arm. The coil was designed to be installed after the marmoset is head fixed in the stereotactic frame via tightening brass screws that electrically close the coil elements around the ear bars. $\boldsymbol{B}$, The tray with the coil removed to show the ear bars, eye bars, and pallet bar (integrated with anesthesia mask). These designs can be 3D printed and are described in detail in Gilbert et al. (2018), along with open-source CAD drawings. C, The data quality of a single raw functional volume (i.e., after conversion to NIfTI format and reorientation, but before any preprocessing; single volume randomly chosen from the fifth marmoset imaged) acquired using the stereotactic frame and radio frequency receive coil shown in $\boldsymbol{A}$ and $\boldsymbol{B}$. $\boldsymbol{D}$, A group temporal SNR map overlaid on the NIH T2-weighted template. The group temporal SNR map shows that the signal in the LFC was relatively high and homogenous across the LFC. Generally, the coil was placed more anteriorly (biasing LFC over posterior visual regions).

discrete functional clusters within the LFC mask. Because hierarchical clustering does not require the specification of a number of clusters, we iterated from 2 through 20 clusters. To estimate the optimum number of clusters for our data, we calculated a silhouette index (MATLAB's "evalclusters" function; higher values indicate more optimal cluster solutions). This method has been applied previously in the RS-fMRI-based functional clustering of macaque prefrontal cortex (Hutchison and Everling, 2014). Based on this analysis, we chose to conduct a seed-based analysis with 7 clusters.

Seed-based cluster analyses. Using the seven discrete functional clusters derived from the normalized group map, seed analyses were con- ducted between each region (i.e., the mean time course within each cluster) and every other voxel in the brain (with the nuisance regressors described above; FSL's FEAT). A group functional connectivity map ( $z$-score map) was then calculated for each of the seven clusters and corrected for multiple comparisons with Gaussian random field theory ( $z>2.3$; cluster significance, $p<0.05$, corrected). The group cluster maps were then projected to surface space with CARET (Van Essen et al., 2001); a surface-based version of the volume template was generously provided by the authors of the NIH marmoset brain template (Liu et al., 2018). 


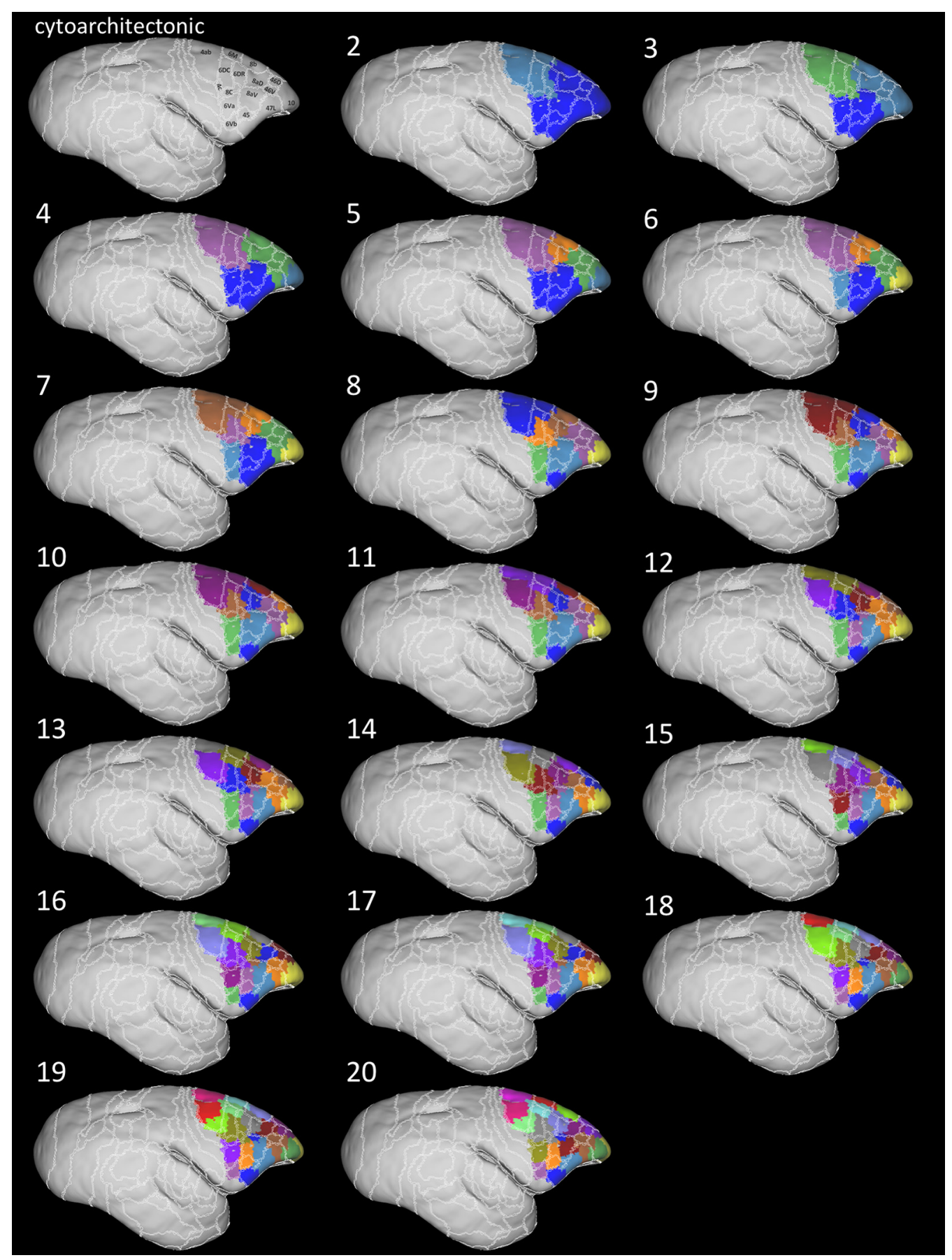

Figure 2. Hierarchical clustering solutions of functional connectivity data within the LFC. Upper left shows cytoarchitectonic boundaries (white lines) and labels of the LFC (Paxinos et al., 2012) displayed on the NIH marmoset brain template surface (Liu et al., 2018). The numbered images display 2 through 20 hierarchical cluster solutions based on the functional connectivity (i.e., time course correlations) within the LFC. These cluster solutions are available for download at https://github.com/NIHMarmosetBrainAtlas/NIH_Marmoset_Atlas_V1/tree/master/v1.1/Results_On_ The Template_Space/Schaeffer_et_al 2019. Colors indicate separate clusters for each cluster solution.

\section{Results}

\section{Hierarchical clustering}

Hierarchical clustering was run on the functional connectivity values between all of the voxels within the LFC (cytoarchitectonically defined areas 10, 46, 47, 9, 8, 45, 6, and 4; Paxinos et al., 2012). As shown in Figure 2, the clustering analysis yielded solutions for 2 through 20 clusters based on the functional connectivity data. To determine a potential optimum number of clusters, we conducted a silhouette analysis, which demonstrated an optimum 11 clusters. The plot of these values, however, sug- gests that the silhouette values start to level off after 7 clusters and remain relatively stable up to 20 clusters (Fig. 3). Given that hierarchical clustering simply subdivides existing clusters as the cluster number increases (i.e., all cluster solutions are subsets of the two-cluster solution; Fig. 3B), we also considered whether the seed-based connectivity maps of the subclusters provided additional information when choosing the optimal cluster number of seven. Given that right and left hemispheres yielded similarly placed clusters (Fig. 4), we report results from the right hemisphere only. 


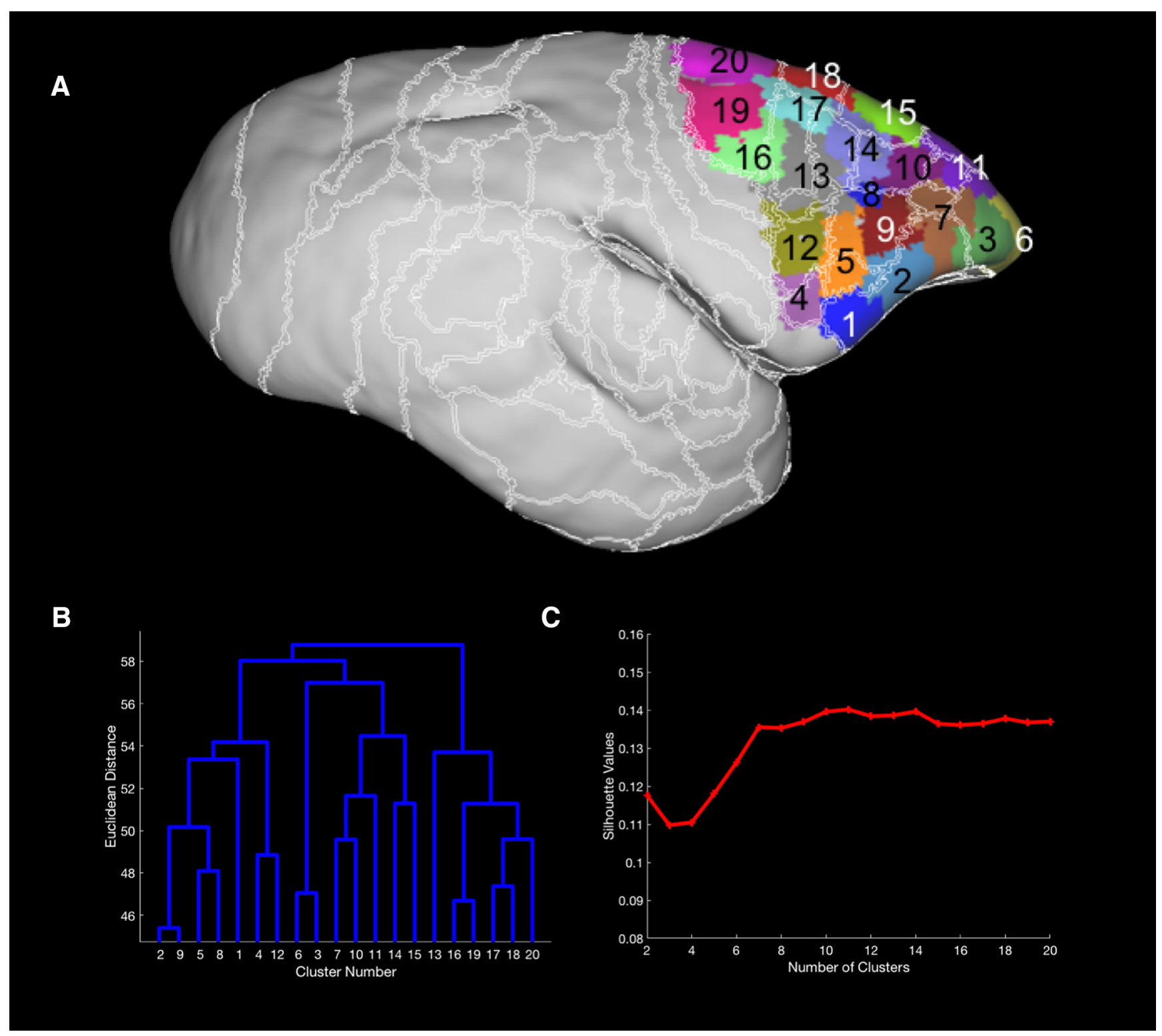

Figure 3. Hierarchical clustering dendrogram and silhouette analysis. $\boldsymbol{A}$, The 20-cluster solution displayed on a surface model (from Fig. 2). The cluster numbers correspond to the $x$-axis in $\boldsymbol{B}$, which shows the dendrogram of the 20 cluster-solution. C, Silhouette values for each cluster solution, with higher values demonstrating a more optimal cluster solution.

\section{Seed-based cluster analyses}

Mean time courses were extracted from each of the seven clusters, and functional connectivity was calculated with each voxel across the rest of the brain. As shown on surface maps in Figure 5 (Fig. 6 for subcortical regions), seven distinct functional connectivity maps emerged (albeit with some regions of overlap). The most rostral cluster (cluster 6) corresponded well to area 10 and showed connectivity that was largely constrained to prefrontal cortex (for specifically labeled regions, see Fig. 5). Cluster 3 corresponded to areas $46 \mathrm{D}$ and $46 \mathrm{~V}$, the anterior portion of $47 \mathrm{~L}$, and the anterior portion of $8 \mathrm{aD}$. The functional connectivity of cluster 3 extended more caudally than that of cluster 6 into anterior cingulate (areas 32 and 24) and lateral temporal areas (temporoparieto-occipital association area; parietal area PGa). Similarly, cluster 4 included multiple cytoarchitectonic areas $(8 \mathrm{~b}, 6 \mathrm{DR}$, and the posterior half of $8 \mathrm{aD}$ ) and showed strong functional connectivity with anterior and midcingulate areas (24, 32, and 23), as well as the temporo-parieto-occipital association area, lateral in- traparietal area, and temporoparietal transitional area. Cluster 1 included $8 \mathrm{aV}, 45$, and the majority of $47 \mathrm{~L}$, which was clearly delineated from the cytoarchitectonic border of area $6 \mathrm{~V}$, which was almost entirely covered by cluster 2 . Clusters 1 and 2 generally showed similar patterns of connectivity, but cluster 1 showed greater connectivity with the temporo-parieto-occipital association area and temporoparietal transitional area. Cluster 5 encompassed areas $6 \mathrm{DC}, 8 \mathrm{C}$, and $4 \mathrm{C}$ and the posterior portion of $6 \mathrm{DR}$, and was connected most strongly to area $4 \mathrm{ab}$, the midcingulate, lateral intraparietal area, and the temporoparietal transitional area. Cluster 7 encompassed area $4 \mathrm{ab}$, but also included area 6DC and $6 \mathrm{M}$ and was most strongly connected to the lateral temporal, midcingulate, and parietal areas.

\section{Discussion}

In this study, we delineated intrinsic functional boundaries of marmoset LFC using ultra-high field RS-fMRI data and a datadriven hierarchical clustering analysis approach. The results 


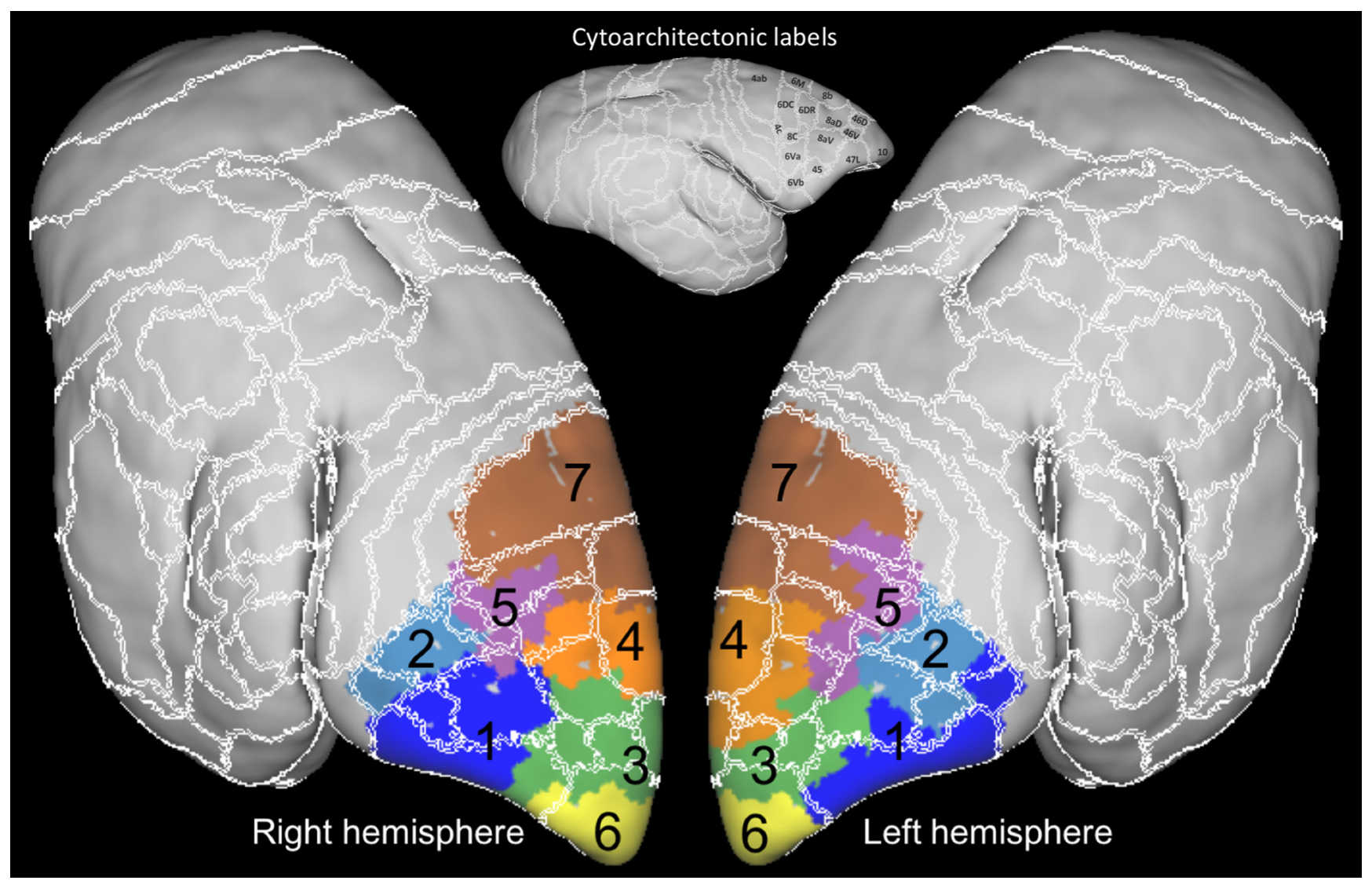

Figure 4. Seven-cluster solutions, conducted separately for each hemisphere. Colors correspond to separate clusters in each hemisphere. When conducting the hierarchical clustering analysis on each hemisphere independently, the spatial distributions are quite similar, suggesting reliability. Note that the left-hemisphere results are displayed on a right-hemisphere surface model ( $x$ direction swapped), as a left-hemisphere surface model was not created. Cytoarchitectonic labels are shown on a lateral surface at the top of the figure for reference.

demonstrated seven distinct functional clusters within LFC, with several of the clusters overlapping well with cytoarchitectonic LFC boundaries (e.g., areas $6 \mathrm{Va}, 6 \mathrm{Vb}$, and 10 ), whereas other functional clusters were not constrained by the cytoarchitectonic boundaries (e.g., those spanning areas 46, 47, 8, and 45). Functional connectivity of these clusters with the rest of the brain was also found to be distinct, with seven patterns of functional connectivity that were organized in a rostrocaudal pattern consonant to that found in humans and macaques (Goulas et al., 2012; Hutchison and Everling, 2014). Overall, these results support the view that marmosets are a promising preclinical modeling species for studying LFC function in healthy and diseased states.

To determine the functional boundaries of LFC, we applied a hierarchical clustering approach to the groupwise functional connectivity values within marmoset LFC (i.e., voxels within cytoarchitectonically defined LFC areas $10,46,47,9,8,45,6$, and 4; Paxinos et al., 2012). As shown in Figure 2, this analysis yielded 2 through 20 cluster solutions in LFC. Although we found the optimal cluster solution to include seven clusters (for silhouette values, see Fig. $3 C$ ), we believe that valuable information exists among other cluster solutions as well. For example, the twocluster solution seemed to clearly demarcate lateral frontal and lateral prefrontal cortex, showing what likely corresponds to a caudal "sensorimotor" cluster and a dorsal "prefrontal" cluster. As these clusters were further subdivided (Fig. 3B), we found that potentially valuable functional boundaries were revealed with each iteration. With cluster solutions above seven, however, we found that further subclustering did not yield sufficiently distinct functional connectivity maps with the rest of the brain (despite similar or even higher silhouette values; Fig. 3). Even with the seven-cluster solution, the lateral surface maps of clusters numbered 4 and 5 were strikingly similar and showed differences only on the medial surface (Fig. 5). Based on these considerations, we chose a seven-cluster cutoff for this study, but as future studies leverage these RS-fMRI based functional boundaries, the optimal cluster solution may depend on scientific question at hand.

The patterns of functional connectivity between mean time courses from the seven clusters and voxel time courses across the rest of the brain (Fig. 5) suggest a LFC network topology that is generally organized along a rostrocaudal gradient (Petrides, 2005; Passingham and Wise, 2012). This pattern corresponds well to what has been reported previously in humans and macaques (for cluster-based patterns of activation in both humans and macaques, see Sallet et al., 2013). For example, when comparing connectivity of the present data (Fig. 5) between rostral clusters (i.e., clusters 1,3 , and 6 ) and caudal clusters (i.e., 4,5 , and 7), it is clear that the rostral clusters have greater connectivity with the anterior portion of cingulate and lateral temporal areas, whereas the caudal clusters have greater connectivity with midcingulate and parietal regions.

The rostrocaudal functional LFC network topology shown here is intriguing from a translational perspective, as it suggests evolutionary preservation of the functional organization of LFC in New World marmosets. Indeed, histochemical tracing studies of marmosets suggest that the structural connectivity of LFC is arranged along the same general rostrocaudal gradient as in humans and macaques (Roberts et al., 2007; Majka et al., 2016). When comparing studies of human and macaque functional 


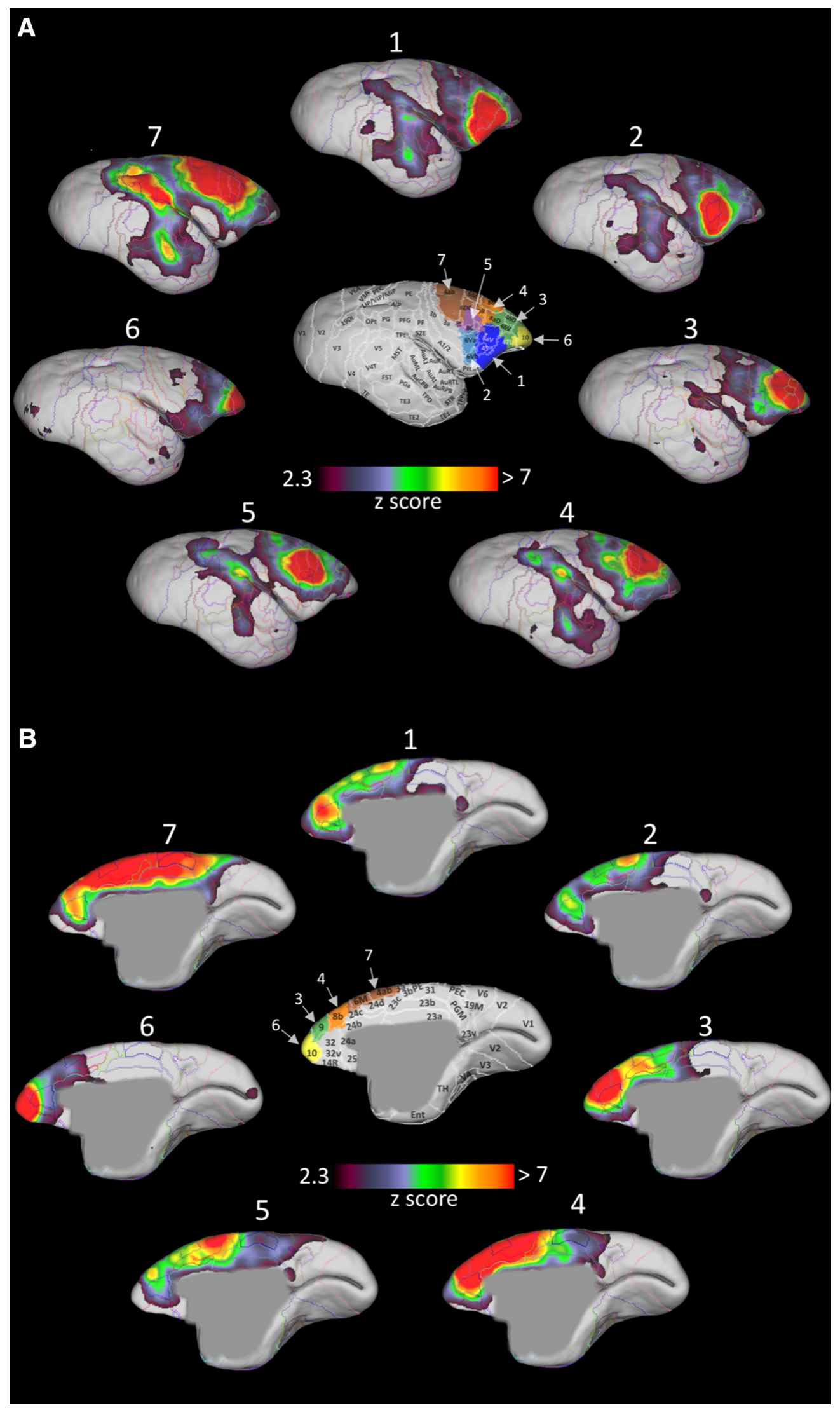

Figure 5. Whole-brain connectivity of LFC clusters. A, The labeled lateral surface (Paxinos et al., 2012; Liu et al., 2018) in the center and the resultant connectivity maps ( $z$ transformed) surrounding the cluster image (numbers in the center correspond to surrounding functional connectivity maps). $\boldsymbol{B}$, The same maps, but on the medial surface. 


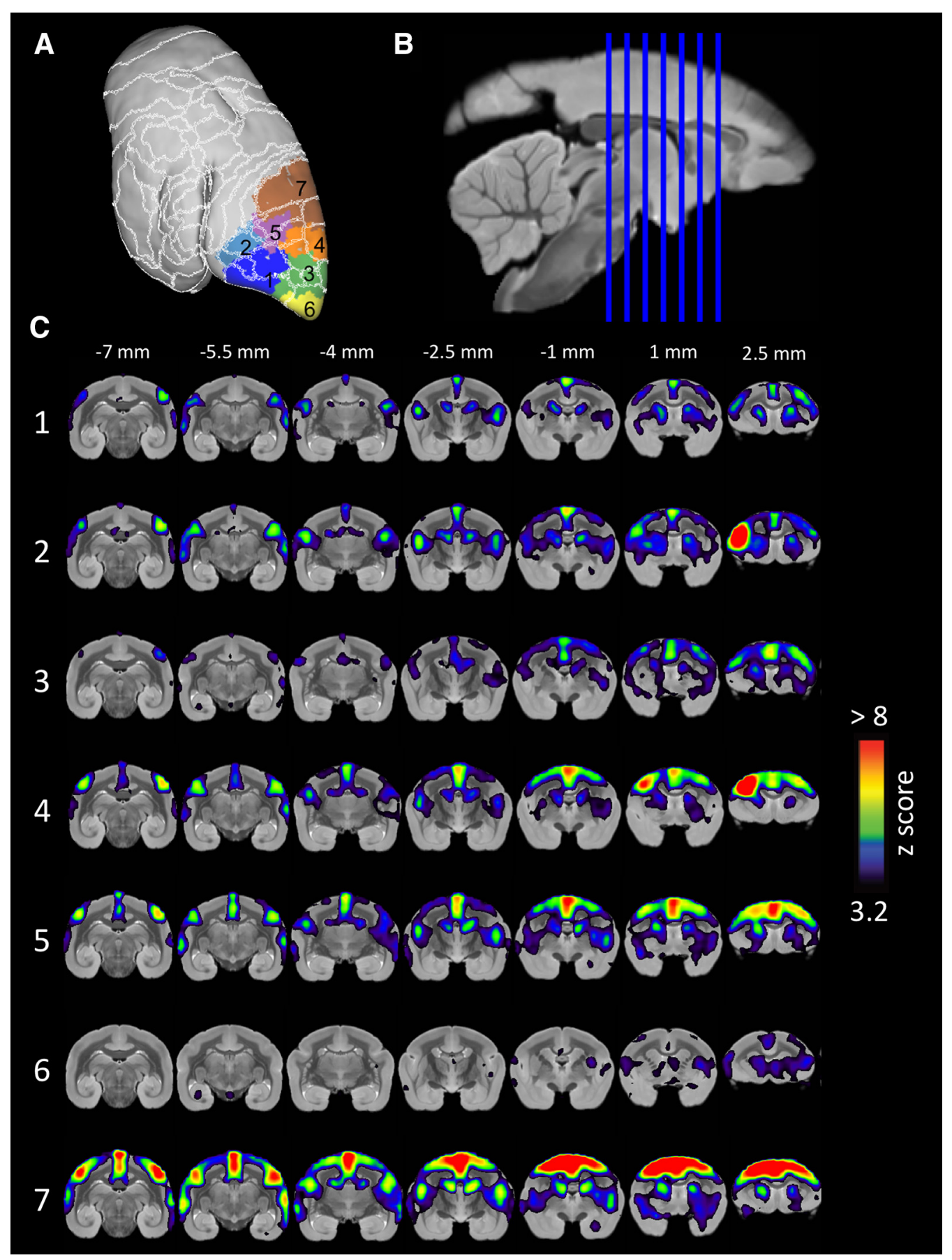

Figure 6. Subcortical connectivity of the seven functional clusters. $\boldsymbol{A}$, The seven-cluster solution (same as in Fig. 4A). $\boldsymbol{B}$, Slice selections (blue lines) against a sagittal slice. $\boldsymbol{C}$, The same slices with functional connectivity values overlaid (same results as shown in Fig. 4 , but displayed volumetrically to show subcortical connectivity). The numbers to the left of the slices in $\boldsymbol{C}$ correspond to the cluster numbers shown in $\boldsymbol{A}$. The numbers above the slices correspond to the distance (in millimeters) from the anterior commissure.

connectivity to the present marmoset data, there are some striking similarities in both cluster location within LFC and the corresponding functional connectivity with the rest of the brain. For instance, Goulas et al. (2012; humans), Sallet et al. (2013; humans and macaques, based on diffusion tensor imaging clustering), and Hutchison and Everling (2014; macaques) all report the following: (1) a rostral cluster corresponding well to area 10 (shown by cluster 6 here; Fig. 5), (2) caudal clusters corresponding to areas $8 \mathrm{aD} / 8 \mathrm{aV}$ (split by clusters 4 and 1 here, and corresponding to areas $8 \mathrm{aD} / 8 \mathrm{aV}$, respectively), and (3) dorsal clusters corresponding to areas $8 \mathrm{~b}$ and extending into area 6 . The Goulas et al.
(2012; humans) and Hutchison and Everling (2014; macaques) studies also show ventral clusters corresponding areas 45 and 47 (cluster 1 here).

Although generally consistent, there are some notable differences between these reported functional boundaries in Old World primates and our present data; for instance, these studies (Goulas et al., 2012; Sallet et al., 2013; Hutchison and Everling, 2014) also show clusters corresponding to area $9 / 46$ (shown by cluster 3 here), but area 46 seems to subcluster into three discrete (and more extensive) clusters, especially in humans. This is not surprising given the relative size of area 46 in marmosets (e.g., size 
of cytoarchitectonic area 46 relative to area 8) compared to Old World primate species (Petrides, 2005; Paxinos et al., 2012). Additionally, areas 9 and 46 have been shown to further subdivide in humans (Rajkowska and Goldman-Rakic, 1995). In area 10 (cluster 6 here), we found that the patterns of connectivity were more similar to that of macaques than to humans (Sallet et al., 2013), with the connectivity largely constrained to frontal cortex. This pattern fits well with recent evidence suggesting that there is not a clear functional connectivity "fingerprint" supporting a homogolous area 10 in macaques (Neubert et al., 2014). It is important to take heed of these differences (e.g., differences in areas 46 or 10 ), as these disparities may have implications for the suitability of the marmoset as a nonhuman primate comparator of human cognition. Given that the density of pyramidal cells in human prefrontal cortex is approximately four times that in marmosets (e.g., area 10) and the marmoset granular prefrontal cortex only occupies $\sim 9 \%$ of the total brain volume (whereas the human granular prefrontal cortex is $\sim 30 \%$ of the total volume; for review, see Elston, 2003), there are clear morphological differences that are likely reflected in cognitive ability, such as working memory.

Although the functional clusters of LFC are described above with reference to cytoarchitectonic boundaries, these boundaries do not strictly overlap in our data. When viewed on the medial wall (Fig. $5 B$ ), the functional boundaries appear to overlap well with the areas 10,9 , and $8 \mathrm{~b}$, with areas $6 \mathrm{M}$ and $4 \mathrm{ab}$ grouped as one cluster. When viewed on the lateral wall (Fig. $5 \mathrm{~A}$ ), however, it is clear that cluster 3 subsumes areas $46 \mathrm{D}$ and $46 \mathrm{~V}$, the anterior portion of $47 \mathrm{~L}$, and the anterior portion of $8 \mathrm{aD}$. Similarly, cluster 4 included area $8 \mathrm{~b}, 6 \mathrm{DR}$, and the posterior half of $8 \mathrm{aD}$. Cluster 1 included $8 \mathrm{aV}, 45$, and the majority of $47 \mathrm{~L}$, which was clearly delineated from the cytoarchitectonic border of area 6V, which was almost entirely covered by cluster 2 . Cluster 7 encompassed area $4 \mathrm{ab}$, but also included area $6 \mathrm{DC}$ and $6 \mathrm{M}$; it is interesting, however, that these functional boundaries seem to overlap better with cytoarchitectonic boundaries at higher cluster solutions (Fig. 2). The intermingling of functional and cytoarchitectonic boundaries has also been shown to occur in Old World primates (Sallet et al., 2013; Hutchison and Everling, 2014) and is consistent with evidence suggesting that prefrontal cortex function relies on the interaction of regions, rather than subregions per se (Miller and Cohen, 2001). A distinct advantage of studying the prefrontal cortex of the New World marmoset brain is that it is lissencephalic, which makes it amenable for laminar electrophysiology or two-photon calcium imaging (i.e., allows for perpendicular electrode/lens placement). This is in contrast to macaques, where the arcuate and principle sulci prevent such recordings across the entirety of prefrontal cortex (Bastos et al., 2018). As such, we expect that further details regarding the functional boundaries and behavioral implications of those boundaries will be elucidated as marmoset neuroscience research continues to burgeon. For example, the existence of an area 44 homolog (not identified here, but which has been identified in macaques; Petrides, 2005; Goulas et al., 2017) may be of particular intrigue for those interested in the evolution of language regions. It seems plausible that our data do not have sufficient spatial resolution to detect a cluster corresponding to area 44 (see 20-cluster solution; Fig. 3A), as the homolog would be quite small in marmosets. As suggested by electrophysiology, however, it could also be the case that vocalization in the marmoset is represented more widely across LFC (i.e., lateral frontal recordings during antiphonal calling; Fig. 4; Miller et al., 2015).
Understanding the network topology of marmoset frontal cortex is critical for the development of preclinical models of human brain diseases. Although the structural boundaries of LFC were described in marmosets at the start of the 20th century (Brodmann, 1909) and refined more recently (Paxinos et al., 2012), the broad functional boundaries of marmoset LFC have yet to be delineated (although lesion, electrophysiology, and fMRI studies have been informative in this regard; Hung et al., 2015; Miller et al., 2015; Shiba et al., 2015; Ghahremani et al., 2017). Here, we demonstrate that the functional network topology of marmoset LFC can be subdivided into discrete functional subregions using RS-fMRI, a noninvasive measure. We expect that these functional boundaries will be useful as models of neuropsychiatric diseases of marmoset are developed (Oikonomidis et al., 2017).

\section{References}

Avants BB, Tustison NJ, Song G, Cook PA, Klein A, Gee JC (2011) A reproducible evaluation of ANTs similarity metric performance in brain image registration. Neuroimage 54:2033-2044. CrossRef Medline

Bastos AM, Loonis R, Kornblith S, Lundqvist M, Miller EK (2018) Laminar recordings in frontal cortex suggest distinct layers for maintenance and control of working memory. Proc Natl Acad Sci U S A 115:1117-1122. CrossRef Medline

Brodmann K (1909) Vergleichende Lokalisationslehre der Grosshirnrinde. Leipzig, Germany: Johann Ambrosius Barth.

Cohen AL, Fair DA, Dosenbach NU, Miezin FM, Dierker D, Van Essen DC, Schlaggar BL, Petersen SE (2008) Defining functional areas in individual human brains using resting functional connectivity MRI. Neuroimage 41:45-57. CrossRef Medline

Cox RW (1996) AFNI: software for analysis and visualization of functional magnetic resonance neuroimages. Comput Biomed Res 29:162-173. CrossRef Medline

Elston GN (2003) Cortex, cognition, and the cell: new insights into the pyramidal neuron and prefrontal function. Cereb Cortex 13:1124-1138. CrossRef Medline

Fuster JM (2001) The prefrontal cortex-an update: time is of the essence. Neuron 30:319-333. CrossRef Medline

Ghahremani M, Hutchison RM, Menon RS, Everling S (2017) Frontoparietal functional connectivity in the common marmoset. Cereb Cortex 27: 3890-3905. Medline

Gilbert KM, Schaeffer DJ, Gati JS, Klassen LM, Everling S, Menon RS (2018) Open-source hardware designs for MRI of mice, rats, and marmosets: integrated animal holders and radiofrequency coils. J Neurosci Methods 312:65-72. CrossRef Medline

Goulas A, Uylings HB, Stiers P (2012) Unravelling the intrinsic functional organization of the human lateral frontal cortex: a parcellation scheme based on resting state fMRI. J Neurosci 32:10238-10252. CrossRef Medline

Goulas A, Stiers P, Hutchison RM, Everling S, Petrides M, Margulies DS (2017) Intrinsic functional architecture of the macaque dorsal and ventral lateral frontal cortex. J Neurophysiol 117:1084-1099. CrossRef Medline

Hung CC, Yen CC, Ciuchta JL, Papoti D, Bock NA, Leopold DA, Silva AC (2015) Functional MRI of visual responses in the awake, behaving marmoset. Neuroimage 120:1-11. CrossRef Medline

Hutchison RM, Everling S (2014) Broad intrinsic functional connectivity boundaries of the macaque prefrontal cortex. Neuroimage 88:202-211. CrossRef Medline

Kishi N, Sato K, Sasaki E, Okano H (2014) Common marmoset as a new model animal for neuroscience research and genome editing technology. Dev Growth Differ 56:53-62. CrossRef Medline

Li X, Morgan PS, Ashburner J, Smith J, Rorden C (2016) The first step for neuroimaging data analysis: DICOM to NIfTI conversion. J Neurosci Methods 264:47-56. CrossRef Medline

Liu C, Ye FQ, Yen CC, Newman JD, Glen D, Leopold DA, Silva AC (2018) A digital 3D atlas of the marmoset brain based on multi-modal MRI. Neuroimage 169:106-116. CrossRef Medline

Majka P, Chaplin TA, Yu HH, Tolpygo A, Mitra PP, Wójcik DK, Rosa MG (2016) Towards a comprehensive atlas of cortical connections in a pri- 
mate brain: mapping tracer injection studies of the common marmoset into a reference digital template. J Comp Neurol 524:2161-2181. CrossRef Medline

Miller CT, Thomas AW, Nummela SU, de la Mothe LA (2015) Responses of primate frontal cortex neurons during natural vocal communication. J Neurophysiol 114:1158-1171. CrossRef Medline

Miller EK, Cohen JD (2001) An integrative theory of prefrontal cortex function. Annu Rev Neurosci 24:167-202. CrossRef Medline

Neubert F-X, Mars RB, Thomas AG, Sallet J, Rushworth MFS (2014) Comparison of human ventral frontal cortex areas for cognitive control and language with areas in monkey frontal cortex. Neuron 81:700-713. CrossRef Medline

Oikonomidis L, Santangelo AM, Shiba Y, Clarke FH, Robbins TW, Roberts AC (2017) A dimensional approach to modeling symptoms of neuropsychiatric disorders in the marmoset monkey. Dev Neurobiol 77:328353. CrossRef Medline

Okano H, Mitra P (2015) Brain-mapping projects using the common marmoset. Neurosci Res 93:3-7. CrossRef Medline

Okano H, Sasaki E, Yamamori T, Iriki A, Shimogori T, Yamaguchi Y, Kasai K, Miyawaki A (2016) Brain/MINDS: a Japanese national brain project for marmoset neuroscience. Neuron 92:582-590. CrossRef Medline

Park JE, Zhang XF, Choi SH, Okahara J, Sasaki E, Silva AC (2016) Generation of transgenic marmosets expressing genetically encoded calcium indicators. Sci Rep 6:34931. CrossRef Medline

Passingham RE, Wise SP (2012) The neurobiology of the prefrontal cortex. New York: Oxford UP.

Paxinos G, Watson C, Petrides M, Rosa M, Tokuno H (2012) The marmoset brain in stereotaxic coordinates. London, UK: Elsevier.

Peterson J, Chaddock R, Dalrymple B, Van Sas F, Gilbert KM, Klassen LM, Gati JS, Handler WB, Chronik BA (2018) Development of a gradient and shim insert system for marmoset imaging at 9.4 T. Paper presented at 26th Annual Meeting of International Society for Magnetic Resonance in Medicine, Paris, June.

Petrides M (2005) Lateral prefrontal cortex: architectonic and functional organization. Philos Trans R Soc B Biol Sci 360:781-795. CrossRef

Rajkowska G, Goldman-Rakic PS (1995) Cytoarchitectonic definition of prefrontal areas in the normal human cortex: II. variability in locations of areas 9 and 46 and relationship to the talairach coordinate system. Cereb Cortex 5:323-337. CrossRef Medline

Reser DH, Burman KJ, Yu HH, Chaplin TA, Richardson KE, Worthy KH, Rosa MG (2013) Contrasting patterns of cortical input to architectural subdivisions of the area 8 complex: a retrograde tracing study in marmoset monkeys. Cereb Cortex 23:1901-1922. CrossRef Medline
Reser DH, Majka P, Snell S, Chan JM, Watkins K, Worthy K, Quiroga MD, Rosa MG (2017) Topography of claustrum and insula projections to medial prefrontal and anterior cingulate cortices of the common marmoset (Callithrix jacchus). J Comp Neurol 525:1421-1441. CrossRef Medline

Roberts AC, Tomic DL, Parkinson CH, Roeling TA, Cutter DJ, Robbins TW, Everitt BJ (2007) Forebrain connectivity of the prefrontal cortex in the marmoset monkey (Callithrix jacchus): an anterograde and retrograde tract-tracing study. J Comp Neurol 502:86-112. CrossRef Medline

Sallet J, Mars RB, Noonan MP, Neubert FX, Jbabdi S, O'Reilly JX, Filippini N, Thomas AG, Rushworth MF (2013) The organization of dorsal frontal cortex in humans and macaques. J Neurosci 33:12255-12274. CrossRef Medline

Sasaki E, Suemizu H, Shimada A, Hanazawa K, Oiwa R, Kamioka M, Tomioka I, Sotomaru Y, Hirakawa R, Eto T, Shiozawa S, Maeda T, Ito M, Ito R, Kito C, Yagihashi C, Kawai K, Miyoshi H, Tanioka Y, Tamaoki N, et al. (2009) Generation of transgenic non-human primates with germline transmission. Nature 459:523-527. CrossRef Medline

Shenton ME, Dickey CC, Frumin M, McCarley RW (2001) A review of MRI findings in schizophrenia. Schizophr Res 49:1-52. Medline

Shiba Y, Kim C, Santangelo AM, Roberts AC (2015) Lesions of either anterior orbitofrontal cortex or ventrolateral prefrontal cortex in marmoset monkeys heighten innate fear and attenuate active coping behaviors to predator threat. Front Syst Neurosci 8:250. Medline

Smith SM, Jenkinson M, Woolrich MW, Beckmann CF, Behrens TE, Johansen-Berg H, Bannister PR, De Luca M, Drobnjak I, Flitney DE, Niazy RK, Saunders J, Vickers J, Zhang Y, De Stefano N, Brady JM, Matthews PM (2004) Advances in functional and structural MR image analysis and implementation as FSL. Neuroimage 23:S208-S219. CrossRef Medline

Tanji J, Hoshi E (2008) Role of the lateral prefrontal cortex in executive behavioral control. Physiol Rev 88:37-57. CrossRef Medline

Tomioka I, Nogami N, Nakatani T, Owari K, Fujita N, Motohashi H, Takayama O, Takae K, Nagai Y, Seki K (2017) Generation of transgenic marmosets using a tetracyclin-inducible transgene expression system as a neurodegenerative disease model. Biol Reprod 97:772-780. CrossRef Medline

Van Essen DC, Drury HA, Dickson J, Harwell J, Hanlon D, Anderson CH (2001) An integrated software suite for surface-based analyses of cerebral cortex. J Am Med Informatics Assoc 8:443-459. CrossRef

Yeterian EH, Pandya DN, Tomaiuolo F, Petrides M (2012) The cortical connectivity of the prefrontal cortex in the monkey brain. Cortex 48:58-81. Medline 